\title{
On the Equivalence of Module Categories over a Group-Theoretical Fusion Category
}

\author{
Sonia NATALE
}

Facultad de Matemática, Astronomía, Física y Computación, Universidad Nacional de Córdoba, CIEM-CONICET, Córdoba, Argentina

E-mail: natale@famaf.unc.edu.ar

URL: http://www. famaf . unc.edu.ar/ natale/

Received April 28, 2017, in final form June 14, 2017; Published online June 17, 2017

https://doi.org/10.3842/SIGMA.2017.042

\begin{abstract}
We give a necessary and sufficient condition in terms of group cohomology for two indecomposable module categories over a group-theoretical fusion category $\mathcal{C}$ to be equivalent. This concludes the classification of such module categories.
\end{abstract}

Key words: fusion category; module category; group-theoretical fusion category

2010 Mathematics Subject Classification: 18D10; 16T05

\section{Introduction}

Throughout this paper we shall work over an algebraically closed field $k$ of characteristic zero. Let $\mathcal{C}$ be a fusion category over $k$. The notion of a $\mathcal{C}$-module category provides a natural categorification of the notion of representation of a group. The problem of classifying module categories plays a fundamental role in the theory of tensor categories.

Two fusion categories $\mathcal{C}$ and $\mathcal{D}$ are called categorically Morita equivalent if there exists an indecomposable $\mathcal{C}$-module category $\mathcal{M}$ such that $\mathcal{D}^{\text {op }}$ is equivalent as a fusion category to the category $\operatorname{Fun}_{\mathcal{C}}(\mathcal{M}, \mathcal{M})$ of $\mathcal{C}$-module endofunctors of $\mathcal{M}$. This defines an equivalence relation in the class of all fusion categories.

Recall that a fusion category $\mathcal{C}$ is called pointed if every simple object of $\mathcal{C}$ is invertible. A basic class of fusion categories consists of those which are categorically Morita equivalent to a pointed fusion category; a fusion category in this class is called group-theoretical. Group-theoretical fusion categories can be described in terms of finite groups and their cohomology.

The purpose of this note is to give a necessary and sufficient condition in terms of group cohomology for two indecomposable module categories over a group-theoretical fusion category to be equivalent. For this, it is enough to solve the same problem for indecomposable module categories over pointed fusion categories.

Let $\mathcal{C}$ be a pointed fusion category. Then there exist a finite group $G$ and a 3-cocycle $\omega$ on $G$ such that $\mathcal{C} \cong \mathcal{C}(G, \omega)$, where $\mathcal{C}(G, \omega)$ is the category of finite-dimensional $G$-graded vector spaces with associativity constraint defined by $\omega$ (see Section 2.3 for a precise definition). Let $\mathcal{M}$ be an indecomposable right $\mathcal{C}$-module category. Then there exists a subgroup $H$ of $G$ and a 2-cochain $\psi \in C^{2}\left(H, k^{\times}\right)$satisfying

$$
d \psi=\left.\omega\right|_{H \times H \times H},
$$

such that $\mathcal{M}$ is equivalent as a $\mathcal{C}$-module category to the category $\mathcal{M}_{0}(H, \psi)$ of left $A(H, \psi)$ modules in $\mathcal{C}$, where $A(H, \psi)=k_{\psi} H$ is the group algebra of $H$ with multiplication twisted by $\psi[8]$, [1, Example 9.7.2].

The main result of this paper is the following theorem. 
Theorem 1.1. Let $H, L$ be subgroups of $G$ and let $\psi \in C^{2}\left(H, k^{\times}\right)$and $\xi \in C^{2}\left(L, k^{\times}\right)$be 2cochains satisfying condition (1.1). Then $\mathcal{M}_{0}(H, \psi)$ and $\mathcal{M}_{0}(L, \xi)$ are equivalent as $\mathcal{C}$-module categories if and only if there exists an element $g \in G$ such that $H={ }^{g} L$ and the class of the 2-cocycle

$$
\left.\xi^{-1} \psi^{g} \Omega_{g}\right|_{L \times L}
$$

is trivial in $H^{2}\left(L, k^{\times}\right)$.

Here we use the notation ${ }^{g} x=g x g^{-1}$ and ${ }^{g} L=\left\{{ }^{g} x: x \in L\right\}$. The 2-cochain $\psi^{g} \in C^{2}\left(L, k^{\times}\right)$ is defined by $\psi^{g}\left(g_{1}, g_{2}\right)=\psi\left({ }^{g} g_{1},{ }^{g} g_{2}\right)$, for all $g_{1}, g_{2} \in L$, and $\Omega_{g}: G \times G \rightarrow k^{\times}$is given by

$$
\Omega_{g}\left(g_{1}, g_{2}\right)=\frac{\omega\left({ }^{g} g_{1},{ }^{g} g_{2}, g\right) \omega\left(g, g_{1}, g_{2}\right)}{\omega\left({ }^{g} g_{1}, g, g_{2}\right)} .
$$

Observe that [8, Theorem 3.1] states that the indecomposable module categories considered in Theorem 1.1 are parameterized by conjugacy classes of pairs $(H, \psi)$. However, this conjugation relation is not described loc. cit. (compare also with [7] and [1, Section 9.7]).

Consider for instance the case where $\mathcal{C}$ is the category of finite-dimensional representations of the 8-dimensional Kac Paljutkin Hopf algebra. Then $\mathcal{C}$ is group-theoretical. In fact, $\mathcal{C} \cong \mathcal{C}(G, \omega, C, 1)$, where $G \cong D_{8}$ is a semidirect product of the group $L=\mathbb{Z}_{2} \times \mathbb{Z}_{2}$ by $C=\mathbb{Z}_{2}$ and $\omega$ is a certain (nontrivial) 3 -cocycle on $G$ [9]. Let $\xi$ represent a nontrivial cohomology class in $H^{2}\left(L, k^{\times}\right)$. According to the usual conjugation relation among pairs $(L, \psi)$, the result in [8, Theorem 3.1] would imply that the pairs $(L, 1)$ and $(L, \xi)$, not being conjugated under the adjoint action of $G$, give rise to two inequivalent $\mathcal{C}$-module categories. These module categories both have rank one, whence they give rise to non-isomorphic fiber functors on $\mathcal{C}$. However, it follows from [4, Theorem 4.8(1)] that the category $\mathcal{C}$ has a unique fiber functor up to isomorphism. In fact, in this example there exists $g \in G$ such that $\left.\Omega_{g}\right|_{L \times L}$ is a 2-cocycle cohomologous to $\xi$. See Example 3.6.

Certainly, the condition given in Theorem 1.1 and the usual conjugacy relation agree in the case where the 3-cocycle $\omega$ is trivial, and it reduces to the conjugation relation among subgroups when they happen to be cyclic.

As explained in Section 3.1, condition (1.2) is equivalent to the condition that $A(L, \xi)$ and ${ }^{g} A(H, \psi)$ be isomorphic as algebras in $\mathcal{C}$, where $\underline{G} \rightarrow \underline{\text { Aut }}_{\otimes} \mathcal{C}, g \mapsto{ }^{g}()$, is the adjoint action of $G$ on $\mathcal{C}$ (see Lemma 3.2).

Theorem 1.1 can be reformulated as follows.

Theorem 1.2. Two $\mathcal{C}$-module categories $\mathcal{M}_{0}(H, \psi)$ and $\mathcal{M}_{0}(L, \xi)$ are equivalent if and only if the algebras $A(H, \psi)$ and $A(L, \xi)$ are conjugated under the adjoint action of $G$ on $\mathcal{C}$.

Theorem 1.1 is proved in Section 3.3. Our proof relies on the fact that, as happens with group actions on vector spaces, the adjoint action of the group $G$ in the set of equivalence classes of $\mathcal{C}$-module categories is trivial (Lemma 3.1). In the course of the proof we establish a relation between the 2-cocycle in (1.2) and a 2-cocycle attached to $g, \psi$ and $\xi$ in [8] (Remark 3.4 and Lemma 3.5).

We refer the reader to [1] for the main notions on fusion categories and their module categories used throughout. 


\section{Preliminaries and notation}

\section{1}

Let $\mathcal{C}$ be a fusion category over $k$. A (right) $\mathcal{C}$-module category is a finite semisimple $k$-linear abelian category $\mathcal{M}$ equipped with a bifunctor $\bar{\otimes}: \mathcal{M} \times \mathcal{C} \rightarrow \mathcal{M}$ and natural isomorphisms

$$
\mu_{M, X, Y}: M \bar{\otimes}(X \otimes Y) \rightarrow(M \bar{\otimes} X) \bar{\otimes} Y, \quad r_{M}: M \bar{\otimes} \mathbf{1} \rightarrow M,
$$

$X, Y \in \mathcal{C}, M \in \mathcal{M}$, satisfying the following conditions:

$$
\begin{aligned}
& \mu_{M \bar{\otimes} X, Y, Z} \mu_{M, X, Y \otimes Z}\left(\operatorname{id}_{M} \bar{\otimes} a_{X, Y, Z}\right)=\left(\mu_{M, X, Y} \otimes \operatorname{id}_{Z}\right) \mu_{M, X \otimes Y, Z}, \\
& \left(r_{M} \otimes \operatorname{id}_{Y}\right) \mu_{M, \mathbf{1}, Y}=\operatorname{id}_{M} \bar{\otimes} l_{Y},
\end{aligned}
$$

for all $M \in \mathcal{M}, X, Y \in \mathcal{C}$, where $a: \otimes \circ\left(\otimes \times \operatorname{id}_{\mathcal{C}}\right) \rightarrow \otimes \circ\left(\operatorname{id}_{\mathcal{C}} \times \otimes\right)$ and $l: 1 \otimes ? \rightarrow \operatorname{id}_{\mathcal{C}}$, denote the associativity and left unit constraints in $\mathcal{C}$, respectively.

Let $A$ be an algebra in $\mathcal{C}$. Then the category ${ }_{A} \mathcal{C}$ of left $A$-modules in $\mathcal{C}$ is a right $\mathcal{C}$-module category with action $\bar{\otimes}:{ }_{A} \mathcal{C} \times \mathcal{C} \rightarrow \mathcal{C}_{A}$, given by $M \bar{\otimes} X=M \otimes X$ endowed with the left $A$-module structure $\left(m_{M} \otimes \operatorname{id}_{X}\right) a_{A, M, X}^{-1}: A \otimes(M \otimes X) \rightarrow M \otimes X$, where $m_{M}: A \otimes M \rightarrow M$ is the $A$ module structure in $M$. The associativity constraint of ${ }_{A} \mathcal{C}$ is given by $a_{M, X, Y}^{-1}: M \bar{\otimes}(X \otimes Y) \rightarrow$ $(M \bar{\otimes} X) \bar{\otimes} Y$, for all $M \in{ }_{A} \mathcal{C}, X, Y \in \mathcal{C}$.

A $\mathcal{C}$-module functor $\mathcal{M} \rightarrow \mathcal{M}^{\prime}$ between right $\mathcal{C}$-module categories $(\mathcal{M}, \bar{\otimes})$ and $\left(\mathcal{M}^{\prime}, \bar{\otimes}^{\prime}\right)$ is a pair $(F, \zeta)$, where $F: \mathcal{M} \rightarrow \mathcal{M}^{\prime}$ is a functor and $\zeta_{M, X}: F(M \bar{\otimes} X) \rightarrow F(M) \bar{\otimes}^{\prime} X$ is a natural isomorphism satisfying

$$
\begin{aligned}
& \left(\zeta_{M, X} \otimes \operatorname{id}_{Y}\right) \zeta_{M \bar{\otimes} X, Y} F\left(\mu_{M, X, Y}\right)=\mu_{F(M), X, Y}^{\prime} \zeta_{M, X \otimes Y}, \\
& r_{F(M)}^{\prime} \zeta_{M, \mathbf{1}}=F\left(r_{M}\right),
\end{aligned}
$$

for all $M \in \mathcal{M}, X, Y \in \mathcal{C}$.

Let $\mathcal{M}$ and $\mathcal{M}^{\prime}$ be $\mathcal{C}$-module categories. An equivalence of $\mathcal{C}$-module categories $\mathcal{M} \rightarrow \mathcal{M}^{\prime}$ is a $\mathcal{C}$-module functor $(F, \zeta): \mathcal{M} \rightarrow \mathcal{M}^{\prime}$ such that $F$ is an equivalence of categories. If such an equivalence exists, $\mathcal{M}$ and $\mathcal{M}^{\prime}$ are called equivalent $\mathcal{C}$-module categories. A $\mathcal{C}$-module category is called indecomposable if it is not equivalent to a direct sum of two nontrivial $\mathcal{C}$-submodule categories.

Let $\mathcal{M}, \mathcal{M}^{\prime}$ be indecomposable $\mathcal{C}$-module categories. Then $\operatorname{Fun}_{\mathcal{C}}(\mathcal{M}, \mathcal{M})$ is a fusion category with tensor product given by composition of functors and the category $\operatorname{Fun}_{\mathcal{C}}\left(\mathcal{M}, \mathcal{M}^{\prime}\right)$ is an indecomposable module category over $\operatorname{Fun}_{\mathcal{C}}(\mathcal{M}, \mathcal{M})$ in a natural way. If $A$ and $B$ are indecomposable algebras in $\mathcal{C}$ such that $\mathcal{M} \cong{ }_{A} \mathcal{C}$ and $\mathcal{M}^{\prime} \cong{ }_{B} \mathcal{C}$, then $\operatorname{Fun}_{\mathcal{C}}(\mathcal{M}, \mathcal{M})^{\text {op }}$ is equivalent to the fusion category ${ }_{A} \mathcal{C}_{A}$ of $(A, A)$-bimodules in $\mathcal{C}$ and there is an equivalence of ${ }_{A} \mathcal{C}_{A}$-module categories ${ }_{B} \mathcal{C}_{A} \cong \operatorname{Fun}_{\mathcal{C}}\left(\mathcal{M}, \mathcal{M}^{\prime}\right)$, where ${ }_{B} \mathcal{C}_{A}$ is the category of $(B, A)$-bimodules in $\mathcal{C}$.

\section{2}

Let $\mathcal{M}$ be a $\mathcal{C}$-module category. Every tensor autoequivalence $\rho: \mathcal{C} \rightarrow \mathcal{C}$ induces a $\mathcal{C}$-module category structure $\mathcal{M}^{\rho}$ on $\mathcal{M}$ in the form $M \bar{\otimes}^{\rho} X=M \bar{\otimes} \rho(X)$, with associativity constraint

$$
\mu_{M, X, Y}^{\rho}=\mu_{M, \rho(X) \otimes \rho(Y)}\left(\operatorname{id}_{M} \bar{\otimes} \rho_{X, Y}^{2}{ }^{-1}\right): \quad M \bar{\otimes} \rho(X \otimes Y) \rightarrow(M \bar{\otimes} \rho(X)) \bar{\otimes} \rho(Y),
$$

for all $M \in \mathcal{M}, X, Y \in \mathcal{C}$, where $\rho_{X, Y}^{2}: \rho(X) \otimes \rho(Y) \rightarrow \rho(X \otimes Y)$ is the monoidal structure of $\rho$. See [7, Section 3.2].

Suppose that $A$ is an algebra in $\mathcal{C}$. Then $\rho(A)$ is an algebra in $\mathcal{C}$ with multiplication

$$
m_{\rho(A)}=\rho\left(m_{A}\right) \rho_{A, A}^{2}: \quad \rho(A) \otimes \rho(A) \rightarrow \rho(A) .
$$


The functor $\rho$ induces an equivalence of $\mathcal{C}$-module categories ${ }_{\rho(A)} \mathcal{C} \rightarrow\left({ }_{A} \mathcal{C}\right)^{\rho}$ with intertwining isomorphisms

$$
\rho_{M, X}^{2}{ }^{-1}: \rho(M \bar{\otimes} X) \rightarrow \rho(M) \bar{\otimes}^{\rho} X .
$$

\section{3}

Let $G$ be a finite group. Let $X$ be a $G$-module. Given an $n$-cochain $f \in C^{n}(G, X)$ (where $\left.C^{0}(G, M)=M\right)$, the coboundary of $f$ is the $(n+1)$-cochain $d f=d^{n} f \in C^{n+1}(G, X)$ defined by

$$
\begin{aligned}
d^{n} f\left(g_{1}, \ldots, g_{n+1}\right)= & g_{1} \cdot f\left(g_{2}, \ldots, g_{n+1}\right)+\sum_{i=1}^{n} f\left(g_{1}, \ldots, g_{i} g_{i+1}, \ldots, g_{n}\right) \\
& +(-1)^{n+1} f\left(g_{1}, \ldots, g_{n}\right)
\end{aligned}
$$

for all $g_{1}, \ldots, g_{n+1} \in G$. The kernel of $d^{n}$ is denoted $Z^{n}(G, M)$; an element of $Z^{n}(G, M)$ is an $n$-cocycle. We have $d^{n} d^{n-1}=0$, for all $n \geq 1$. The $n$th cohomology group of $G$ with coefficients in $M$ is $H^{n}(G, M)=Z^{n}(G, M) / d^{n-1}\left(C^{n-1}(G, M)\right)$. We shall write $f \equiv f^{\prime}$ when the cochains $f, f^{\prime} \in C^{n}\left(G, k^{\times}\right)$differ by a coboundary.

We shall assume that every cochain $f$ is normalized, that is, $f\left(g_{1}, \ldots, g_{n}\right)=1$, whenever one of the arguments $g_{1}, \ldots, g_{n}$ is the identity. If $H$ is a subgroup of $G$ and $f \in C^{n}\left(H, k^{\times}\right)$, we shall indicate by $f^{g}$ the $n$-cochain in ${ }^{g^{-1}} H$ given by $f^{g}\left(h_{1}, \ldots, h_{n}\right)=f\left({ }^{g} h_{1}, \ldots,{ }^{g} h_{n}\right), h_{1}, \ldots, h_{n} \in H$.

Let $\omega: G \times G \times G \rightarrow k^{\times}$be a 3-cocycle on $G$. Let $\mathcal{C}(G, \omega)$ denote the fusion category of finite-dimensional $G$-graded vector spaces with associativity constraint defined, for all $U, V, W \in$ $\mathcal{C}(G, \omega)$, as

$$
a_{X, Y, Z}((u \otimes v) \otimes w)=\omega^{-1}\left(g_{1}, g_{2}, g_{3}\right) u \otimes(v \otimes w),
$$

for all homogeneous vectors $u \in U_{g_{1}}, v \in V_{g_{2}}, w \in W_{g_{3}}, g_{1}, g_{2}, g_{3} \in G$. Any pointed fusion category is equivalent to a category of the form $\mathcal{C}(G, \omega)$.

A fusion category $\mathcal{C}$ is called group-theoretical if it is categorically Morita equivalent to a pointed fusion category. Equivalently, $\mathcal{C}$ is group-theoretical if and only if there exist a finite group $G$ and a 3-cocycle $\omega: G \times G \times G \rightarrow k^{\times}$such that $\mathcal{C}$ is equivalent to the fusion category $\mathcal{C}(G, \omega, H, \psi)={ }_{A(H, \psi)} \mathcal{C}(G, \omega)_{A(H, \psi)}$, where $H$ is a subgroup of $G$ such that the class of $\left.\omega\right|_{H \times H \times H}$ is trivial and $\psi: H \times H \rightarrow k^{\times}$is a 2-cochain on $H$ satisfying condition (1.1).

Let $\mathcal{C}(G, \omega, H, \psi) \cong \mathcal{C}(G, \omega)_{\mathcal{M}_{0}(H, \psi)}^{*}$ be a group-theoretical fusion category. Then there is a bijective correspondence between equivalence classes of indecomposable $\mathcal{C}(G, \omega, H, \psi)$-module categories and equivalence classes of indecomposable $\mathcal{C}(G, \omega)$-module categories. This correspondence attaches to every indecomposable $\mathcal{C}(G, \omega)$-module category $\mathcal{M}$ the $\mathcal{C}(G, \omega, H, \psi)$-module category

$$
\mathcal{M}(H, \psi)=\operatorname{Fun}_{\mathcal{C}(G, \omega)}\left(\mathcal{M}_{0}(H, \psi), \mathcal{M}\right) .
$$

\section{Indecomposable module categories over $\mathcal{C}(G, \omega)$}

Throughout this section $G$ is a finite group and $\omega: G \times G \times G \rightarrow k^{\times}$is a 3-cocycle on $G$.

\section{1}

Let $g \in G$. Consider the 2-cochain $\Omega_{g}: G \times G \rightarrow k^{\times}$given by

$$
\Omega_{g}\left(g_{1}, g_{2}\right)=\frac{\omega\left({ }^{g} g_{1},{ }^{g} g_{2}, g\right) \omega\left(g, g_{1}, g_{2}\right)}{\omega\left({ }^{g} g_{1}, g, g_{2}\right)} .
$$


For all $g \in G$ we have the relation

$$
d \Omega_{g}=\frac{\omega}{\omega^{g}} .
$$

Let $\mathcal{C}=\mathcal{C}(G, \omega)$ and let $g \in G$. For every object $V$ of $\mathcal{C}$ let ${ }^{g} V$ be the object of $\mathcal{C}$ such that ${ }^{g} V=V$ as a vector space with $G$-grading defined as $\left({ }^{g} V\right)_{x}=V_{g_{x}}, x \in G$. For every $g \in G$, we have a functor $\operatorname{ad}_{g}: \mathcal{C} \rightarrow \mathcal{C}$, given by $\operatorname{ad}_{g}(V)={ }^{g} V$ and $\operatorname{ad}_{g}(f)=f$, for every object $V$ and morphism $f$ of $\mathcal{C}$. Relation (3.1) implies that $\operatorname{ad}_{g}$ is a tensor functor with monoidal structure defined by

$$
\left(\operatorname{ad}_{g}^{2}\right)_{U, V}:{ }^{g} U \otimes{ }^{g} V \rightarrow{ }^{g}(U \otimes V), \quad\left(\operatorname{ad}_{g}^{2}\right)_{U, V}(u \otimes v)=\Omega_{g}\left(h, h^{\prime}\right)^{-1} u \otimes v,
$$

for all $h, h^{\prime} \in G$, and for all homogeneous vectors $u \in U_{h}, v \in V_{h^{\prime}}$.

For every $g, g_{1}, g_{2} \in G$, let $\gamma\left(g_{1}, g_{2}\right): G \rightarrow k^{\times}$be the map defined in the form

$$
\gamma\left(g_{1}, g_{2}\right)(g)=\frac{\omega\left(g_{1}, g_{2}, g\right) \omega\left({ }^{g_{1}} g_{2} g, g_{1}, g_{2}\right)}{\omega\left(g_{1},{ }^{g_{2}} g, g_{2}\right)} .
$$

The following relation holds, for all $g_{1}, g_{2} \in G$ :

$$
\Omega_{g_{1} g_{2}}=\Omega_{g_{1}}^{g_{2}} \Omega_{g_{2}} d \gamma\left(g_{1}, g_{2}\right) .
$$

In this way, ad: $\underline{G} \rightarrow \underline{\operatorname{Aut}}_{\otimes} \mathcal{C}, \operatorname{ad}(g)=\left(\operatorname{ad}_{g}, \operatorname{ad}_{g}^{2}\right)$, gives rise to an action by tensor autoequivalences of $G$ on $\mathcal{C}$ where, for every $g, x \in G, V \in \mathcal{C}(G, \omega)$, the monoidal isomorphisms $\mathrm{ad}_{V}^{2}:{ }^{g}\left(g^{\prime} V\right) \rightarrow{ }^{g g^{\prime}} V$ are given by

$$
\operatorname{ad}_{V}^{2}(v)=\gamma\left(g, g^{\prime}\right)(x) v
$$

for all homogeneous vectors $v \in V_{x}, h \in G$. The equivariantization $\mathcal{C}^{G}$ with respect to this action is equivalent to the category of finite-dimensional representations of the twisted quantum double $D^{\omega} G$ (see [5, Lemma 6.3]).

For each $g \in G$, and for each $\mathcal{C}$-module category $\mathcal{M}$, let $\mathcal{M}^{g}$ denote the module category induced by the functor $\operatorname{ad}_{g}$ as in Section 2.2. Recall that the action of $\mathcal{C}$ on $\mathcal{M}^{g}$ is defined by $M \bar{\otimes}{ }^{g} V=M \bar{\otimes}\left({ }^{g} V\right)$, for all objects $V$ of $\mathcal{C}$.

Lemma 3.1. Let $g \in G$ and let $\mathcal{M}$ be a $\mathcal{C}$-module category. Then $\mathcal{M}^{g} \cong \mathcal{M}$ as $\mathcal{C}$-module categories.

Proof. For each $g \in G$, let $\{g\}$ denote the object of $\mathcal{C}$ such that $\{g\}=k$ with degree $g$. In what follows, by abuse of notation, we identify $\{g\} \otimes\{h\}$ and $\{g h\}, g, h \in G$, by means of the canonical isomorphisms of vector spaces.

Let $R_{g}: \mathcal{M}^{g} \rightarrow \mathcal{M}$ be the functor defined by the right action of $\{g\}: R_{g}(M)=M \bar{\otimes}\{g\}$. Consider the natural isomorphism $\zeta: R_{g} \circ \bar{\otimes}^{g} \rightarrow \bar{\otimes} \circ\left(R_{g} \times \mathrm{id}_{\mathcal{C}}\right)$, defined as

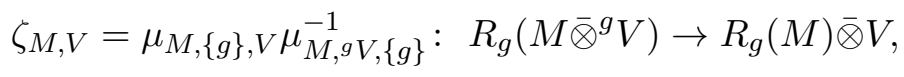

for all objects $M$ of $\mathcal{M}$ and $V$ of $\mathcal{C}$, where $\mu$ is the associativity constraint of $\mathcal{M}$.

The functor $R_{g}$ is an equivalence of categories with quasi-inverse given by the functor $R_{g^{-1}}$ : $\mathcal{M} \rightarrow \mathcal{M}^{g}$.

A direct calculation, using the coherence conditions (2.1) and (2.2) for the module category $\mathcal{M}$, shows that $\zeta$ satisfies conditions $(2.3)$ and $(2.4)$. Hence $\left(R_{g}, \zeta\right)$ is a $\mathcal{C}$-module functor. Therefore $\mathcal{M}^{g} \cong \mathcal{M}$ as $\mathcal{C}$-module categories, as claimed.

Lemma 3.2. Let $H$ be a subgroup of $G$ and let $\psi$ be a 2-cochain on $H$ satisfying (1.1). Let $A(H, \psi)$ denote the corresponding indecomposable algebra in $\mathcal{C}$. Then, for all $g \in G,{ }^{g} A(H, \psi) \cong$ $A\left({ }^{g} H, \psi^{g^{-1}} \Omega_{g^{-1}}\right)$ as algebras in $\mathcal{C}$.

Proof. By definition, ${ }^{g} A(H, \psi)=A\left({ }^{g} H, \psi^{g^{-1}}\left(\Omega_{g}^{g^{-1}}\right)^{-1}\right)$. It follows from formula (3.2) that $\left(\Omega_{g}^{g^{-1}}\right)^{-1}$ and $\Omega_{g^{-1}}$ differ by a coboundary. This implies the lemma. 


\section{2}

Let $H, L$ be subgroups of $G$ and let $\psi \in C^{2}\left(H, k^{\times}\right), \xi \in C^{2}\left(L, k^{\times}\right)$, be 2-cochains such that $\left.\omega\right|_{H \times H \times H}=d \psi$ and $\left.\omega\right|_{L \times L \times L}=d \xi$.

Let $B$ be an object of the category ${ }_{A(H, \psi)} \mathcal{C}_{A(L, \xi)}$ of $(A(H, \psi), A(L, \xi))$-bimodules in $\mathcal{C}$. For each $z \in G$, let $\pi_{l}(h): B_{z} \rightarrow B_{h z}$ and $\pi_{r}(s): B_{z} \rightarrow B_{z s}$, denote the linear maps induced by the actions of $h \in H$ and $s \in L$, respectively. Then the following relations hold, for all $h, h^{\prime} \in H$, $s, s^{\prime} \in L:$

$$
\begin{aligned}
& \pi_{l}(h) \pi_{l}\left(h^{\prime}\right)=\omega\left(h, h^{\prime}, z\right) \psi\left(h, h^{\prime}\right) \pi_{l}\left(h h^{\prime}\right), \\
& \pi_{r}\left(s^{\prime}\right) \pi_{r}(s)=\omega\left(z, s, s^{\prime}\right)^{-1} \xi\left(s, s^{\prime}\right) \pi_{r}\left(s s^{\prime}\right), \\
& \pi_{l}(h) \pi_{r}(s)=\omega(h, z, s) \pi_{r}(s) \pi_{l}(h) .
\end{aligned}
$$

Lemma 3.3. Let $g \in G$ and let $B_{g}$ denote the homogeneous component of degree $g$ of $B$. Then the map $\pi: H \cap{ }^{g} L \rightarrow \mathrm{GL}\left(B_{g}\right)$, defined as $\pi(x)=\pi_{r}\left(g^{-1} x\right)^{-1} \pi_{l}(x)$ is a projective representation of $H \cap{ }^{g} L$ with cocycle $\alpha_{g}$ given, for all $x, y \in H \cap{ }^{g} L$, as follows:

$$
\begin{aligned}
\alpha_{g}(x, y)= & \psi(x, y) \xi^{-1}\left(g^{-1} x, g^{-1} y\right) \frac{\omega(x, y, g) \omega\left(x, y g,,^{-1}\left(y^{-1}\right)\right)}{\omega\left(x y g, g^{-1}\left(y^{-1}\right), g^{-1}\left(x^{-1}\right)\right)} d u_{g}(x, y) \\
& \times \frac{\omega\left(g^{-1} y, g^{-1}\left(y^{-1}\right), g^{-1}\left(x^{-1}\right)\right)}{\omega\left(g^{-1} x, g^{-1} y, g^{-1}\left(y^{-1} x^{-1}\right)\right)},
\end{aligned}
$$

where the 1-cochain $u_{g}$ is defined as $u_{g}(x)=\omega\left(x g, g^{-1} x, g^{-1}\left(x^{-1}\right)\right)$.

Proof. It follows from (3.4) that $\pi_{r}(s)^{-1}=\omega\left(z, s, s^{-1}\right) \xi\left(s, s^{-1}\right)^{-1} \pi_{r}\left(s^{-1}\right)$, for all $z \in G, s \in L$. In addition, for all $h, h^{\prime} \in L$, we have the following relation:

$$
\xi\left(h^{\prime-1}, h^{-1}\right) \xi\left(h, h^{\prime}\right)=d f\left(h, h^{\prime}\right) \frac{\omega\left(h^{\prime}, h^{\prime-1}, h^{-1}\right)}{\omega\left(h, h^{\prime}, h^{\prime-1} h^{-1}\right)},
$$

where $f$ is the 1-cochain given by $f(h)=\xi\left(h, h^{-1}\right)$. A straightforward computation, using this relation and conditions (3.3), (3.4) and (3.5), shows that $\pi(x) \pi(y)=\alpha_{g}(x, y) \pi(x y)$, for all $x, y \in H \cap{ }^{g} L$. This proves the lemma.

Remark 3.4. Lemma 3.3 is a version of [8, Proposition 3.2], where it is shown that $B$ is a simple object of ${ }_{A(H, \psi)} \mathcal{C}_{A(L, \xi)}$ if and only if $B$ is supported on a single double coset $H g L$ and the projective representation $\pi$ in the component $B_{g}$ is irreducible.

For all $g \in G, \psi^{g} \Omega_{g}$ is a 2-cochain in ${ }^{g^{-1} H}$ such that $\left.\omega\right|_{g^{-1} H \times{ }^{-1} H \times{ }^{-1} H}=d\left(\psi^{g} \Omega_{g}\right)$. Then the product $\xi^{-1} \psi^{g} \Omega_{g}$ defines a 2-cocycle of ${ }^{g^{-1}} H \cap L$.

Lemma 3.5. The class of the 2-cocycle $\left(\xi^{-1} \psi^{g} \Omega_{g}\right)^{g^{-1}}$ in $H^{2}\left(H \cap{ }^{g} L, k^{\times}\right)$coincides with the class of the 2-cocycle $\alpha_{g}$ in Lemma 3.3 .

Proof. A direct calculation shows that for all $x, y \in G$,

$$
\frac{\omega\left(y, y^{-1}, x^{-1}\right)}{\omega\left(x, y, y^{-1} x^{-1}\right)} \frac{\omega\left({ }^{g} x,{ }^{g} y, g\right) \omega\left({ }^{g} x,{ }^{g} y g, y^{-1}\right)}{\omega\left({ }^{g} x^{g} y g, y^{-1}, x^{-1}\right)}=\Omega_{g}(x, y) d \theta_{g}(x, y),
$$

where the 1-cochain $\theta_{g}$ is defined as $\theta_{g}(x)=\omega\left(g, x, x^{-1}\right)^{-1}$. This implies that $\alpha_{g}^{g} \equiv \xi^{-1} \psi^{g} \Omega_{g}$, as was to be proved. 


\section{3}

In this subsection we give a proof of the main result of this paper.

Proof of Theorem 1.1. Let $H, L$ be subgroups of $G$ and let $\psi \in C^{2}\left(H, k^{\times}\right)$and $\xi \in C^{2}\left(L, k^{\times}\right)$ be 2-cochains satisfying condition (1.1). Let $A(H, \psi), A(L, \xi)$ be the associated algebras in $\mathcal{C}$ and let $\mathcal{M}_{0}(H, \psi), \mathcal{M}_{0}(L, \xi)$ be the corresponding $\mathcal{C}$-module categories.

Let $\mathcal{M}=\mathcal{M}_{0}(L, \xi)$. For every $g \in G$, let $\mathcal{M}^{g}$ denote the module category induced by the autoequivalence $\operatorname{ad}_{g}: \mathcal{C} \rightarrow \mathcal{C}$. The $\mathcal{C}$-module category $\mathcal{M}^{g}$ is equivalent to ${ }_{g} A(L, \xi) \mathcal{C}$. Hence, by Lemma 3.2, $\mathcal{M}^{g} \cong \mathcal{M}_{0}\left({ }^{g} L, \xi^{g^{-1}} \Omega_{g^{-1}}\right)$.

Suppose that there exists an element $g \in G$ such that $H={ }^{g} L$ and the class of the cocycle $\xi^{-1} \psi^{g} \Omega_{g}$ is trivial on $L$. Relation (3.2) implies that $\Omega_{g}^{g^{-1}}=\Omega_{g^{-1}}^{-1}$, and thus the class of $\psi^{-1} \xi^{g^{-1}} \Omega_{g^{-1}}$ is trivial on $H$. Then $\psi=\xi^{g^{-1}} \Omega_{g^{-1}} d f$, for some 1-cochain $f \in C^{1}\left(H, k^{\times}\right)$. Therefore ${ }^{g} A(L, \xi)=A\left(H, \xi^{g^{-1}} \Omega_{g^{-1}}\right) \cong A(H, \psi)$ as algebras in $\mathcal{C}$. Thus we obtain equivalences of $\mathcal{C}$-module categories

$$
\mathcal{M}_{0}(L, \xi) \cong \mathcal{M}_{0}(L, \xi)^{g} \cong{ }_{g}(L, \xi) \mathcal{C} \cong \mathcal{M}_{0}(H, \psi),
$$

where the first equivalence is deduced from Lemma 3.1.

Conversely, suppose that $F: \mathcal{M}_{0}(L, \xi) \rightarrow \mathcal{M}_{0}(H, \psi)$ is an equivalence of $\mathcal{C}$-module categories. Recall that there is an equivalence

$$
\operatorname{Fun}_{\mathcal{C}}\left(\mathcal{M}_{0}(L, \xi), \mathcal{M}_{0}(H, \psi)\right) \cong{ }_{A(H, \psi)} \mathcal{C}_{A(L, \xi)} .
$$

Under this equivalence, the functor $F$ corresponds to an object $B$ of ${ }_{A(H, \psi)} \mathcal{C}_{A(L, \xi)}$ such that there exists an object $B^{\prime}$ of ${ }_{A(L, \xi)} \mathcal{C}_{A(H, \psi)}$ satisfying

$$
B \otimes_{A(L, \xi)} B^{\prime} \cong A(H, \psi),
$$

as $A(H, \psi)$-bimodules in $\mathcal{C}$, and

$$
B^{\prime} \otimes_{A(H, \psi)} B \cong A(L, \xi),
$$

as $A(L, \xi)$-bimodules in $\mathcal{C}$.

Let $\operatorname{FPdim}_{A(H, \psi)} M$ denote the Frobenius-Perron dimension of an object $M$ of ${ }_{A(H, \psi)} \mathcal{C}_{A(H, \psi)}$. Then we have

$$
\operatorname{dim} M=\operatorname{dim} A(H, \psi) \operatorname{FPdim}_{A(H, \psi)} M=|H| \operatorname{FPdim}_{A(H, \psi)} M .
$$

Taking Frobenius-Perron dimensions in both sides of (3.6) and using this relation we obtain that $\operatorname{dim}\left(B \otimes_{A(L, \xi)} B^{\prime}\right)=|H|$.

On the other hand, $\operatorname{dim}\left(B \otimes_{A(H, \psi)} B^{\prime}\right)=\frac{\operatorname{dim} B \operatorname{dim} B^{\prime}}{\operatorname{dim} A(L, \xi)}=\frac{\operatorname{dim} B \operatorname{dim} B^{\prime}}{|L|}$. Thus

$$
\operatorname{dim} B \operatorname{dim} B^{\prime}=|H||L| \text {. }
$$

Since $A(H, \psi)$ is an indecomposable algebra in $\mathcal{C}$, then it is a simple object of ${ }_{A(H, \psi)} \mathcal{C}_{A(H, \psi)}$. Then (3.7) implies that $B$ is a simple object of ${ }_{A(H, \psi)} \mathcal{C}_{A(L, \xi)}$ and $B^{\prime}$ is a simple object of $A(L, \xi) \mathcal{C}_{A(H, \psi)}$.

In view of [8, Proposition 3.2], the support of $B$ is a two sided $(H, L)$-double coset, that is, $B=\bigoplus_{\left(h, h^{\prime}\right) \in H \times L} B_{h g h^{\prime}}$, where $g \in G$ is a representative of the double coset that supports $B$. Moreover, the homogeneous component $B_{g}$ is an irreducible $\alpha_{g}$-projective representation of the group ${ }^{g} L \cap H$, where the 2-cocycle $\alpha_{g}$ satisfies $\alpha_{g} \equiv\left(\xi^{-1} \psi^{g} \Omega_{g}\right)^{g^{-1}}$; see Remark 3.4 and Lemmas 3.3 and 3.5. 
Notice that the actions of $h \in H$ and $h^{\prime} \in L$ induce isomorphisms of vector spaces $B_{g} \cong B_{h g}$ and $B_{g} \cong B_{g h^{\prime}}$. Hence

$$
\operatorname{dim} B=|H g L| \operatorname{dim} B_{g}=\frac{|H||L|}{\left|H \cap{ }^{g} L\right|} \operatorname{dim} B_{g}=\left[H: H \cap{ }^{g} L\right]|L| \operatorname{dim} B_{g} .
$$

In particular, $\operatorname{dim} B \geq|L|$. Reversing the roles of $H$ and $L$, the same argument implies that $\operatorname{dim} B^{\prime} \geq|H|$. Combined with relations (3.8) and (3.9) this implies

$$
|H||L|=\operatorname{dim} B \operatorname{dim} B^{\prime} \geq|H|\left[H: H \cap{ }^{g} L\right]|L| \operatorname{dim} B_{g} .
$$

Hence $\left[H: H \cap{ }^{g} L\right] \operatorname{dim} B_{g}=1$, and therefore $\left[H: H \cap{ }^{g} L\right]=1$ and $\operatorname{dim} B_{g}=1$. The first condition means that $H \subseteq{ }^{g} L$, while the second condition implies that the class of $\alpha_{g}$ is trivial in $H^{2}\left(H \cap{ }^{g} L, k^{\times}\right)$. Since the rank of $\mathcal{M}_{0}(H, \psi)$ equals the index $[G: H]$ and the rank of $\mathcal{M}_{0}(H, \xi)$ equals the index $[G: L]$, then $|H|=|L|$. Thus we get that $H={ }^{g} L$ and that the class of the 2-cocycle (1.2) is trivial in $H^{2}\left(L, k^{\times}\right)$. This finishes the proof of the theorem.

Example 3.6. Let $B_{8}$ be the 8-dimensional Kac Paljutkin Hopf algebra. The Hopf algebra $B_{8}$ fits into an exact sequence

$$
k \longrightarrow k^{C} \longrightarrow B_{8} \longrightarrow k L \longrightarrow k,
$$

where $C=\mathbb{Z}_{2}$ and $L=\mathbb{Z}_{2} \times \mathbb{Z}_{2}$. See [3]. This exact sequence gives rise to mutual actions by permutations

$$
C \stackrel{\triangleleft}{\longleftarrow} \times L \stackrel{\triangleright}{\longrightarrow} L
$$

and compatible cocycles $\tau: L \times L \rightarrow\left(k^{C}\right)^{\times}, \sigma: C \times C \rightarrow\left(k^{L}\right)^{\times}$, such that $B_{8}$ is isomorphic to the bicrossed product $k C^{\tau} \#_{\sigma} k L$. The data $\triangleleft, \triangleright, \sigma$ and $\tau$ are explicitly determined in [4, Proposition 3.11] as follows. Let $C=\left\langle x: x^{2}=1\right\rangle, L=\left\langle z, t: z^{2}=t^{2}=z t z^{-1} t^{-1}=1\right\rangle$. Then $\triangleleft: C \times L \rightarrow C$ is the trivial action of $L$ on $C, \triangleright: C \times L \rightarrow L$ is the action defined by $x \triangleright z=z$ and $x \triangleright t=z t$,

$$
\tau_{x^{n}}\left(z^{i} t^{j}, z^{i^{\prime}} t^{j^{\prime}}\right)=(-1)^{n j i^{\prime}}
$$

for all $0 \leq n, i, i^{\prime}, j, j^{\prime} \leq 1$, and

$$
\sigma_{z^{i} t^{j}}\left(x^{n}, x^{n^{\prime}}\right)=(\sqrt{-1})^{j\left(\frac{n+n^{\prime}-\left\langle n+n^{\prime}\right\rangle}{2}\right)},
$$

for all $0 \leq i, j, n, n^{\prime} \leq 1$, where $\left\langle n+n^{\prime}\right\rangle$ denotes the remainder of $n+n^{\prime}$ in the division by 2 . Here we use the notation $\tau\left(a, a^{\prime}\right)(y)=: \tau_{y}\left(a, a^{\prime}\right)$ and, similarly, $\sigma\left(y, y^{\prime}\right)(a)=: \sigma_{a}\left(y, y^{\prime}\right), a, a^{\prime} \in L$, $y, y^{\prime} \in C$.

In view of [9, Theorem 3.3.5] (see [6, Proposition 4.3]), the fusion category of finite-dimensional representations of $B_{8}^{\mathrm{op}} \cong B_{8}$ is equivalent to the category $\mathcal{C}(G, \omega, L, 1)$, where $G=L \rtimes C$ is the semidirect product with respect to the action $\triangleright$, and $\omega$ is the 3-cocycle arising from the pair $(\tau, \sigma)$ under one of the maps of the so-called Kac exact sequence associated to the matched pair.

In this example $G$ is isomorphic to the dihedral group $D_{8}$ of order 8 . The 3 -cocycle $\omega$ is determined by the formula

$$
\omega\left(x^{n} z^{i} t^{j}, x^{n^{\prime}} z^{i^{\prime}} t^{j^{\prime}}, x^{n^{\prime \prime}} z^{i^{\prime \prime}} t^{j^{\prime \prime}}\right)=\tau_{x^{n}}\left(z^{i^{\prime}} t^{j^{\prime}}, x^{n^{\prime}} \triangleright z^{i^{\prime \prime}} t^{j^{\prime \prime}}\right) \sigma_{z^{i^{\prime \prime}}} t^{j^{\prime \prime}}\left(x^{n}, x^{n^{\prime}}\right),
$$

for all $0 \leq i, j, i^{\prime}, j^{\prime}, i^{\prime \prime}, j^{\prime \prime}, n, n^{\prime}, n^{\prime \prime} \leq 1$ 
Notice that $\left.\omega\right|_{L \times L \times L}=1$. Hence, for every 2-cocycle $\xi$ on $L$, the pair $(L, \xi)$ gives rise to an indecomposable $\mathcal{C}$-module category $\mathcal{M}(L, \xi)$. Formula (3.10) implies that $\left.\Omega_{x}\right|_{L \times L}$ is given by

$$
\Omega_{x}\left(z^{i} t^{j}, z^{i^{\prime}} t^{j^{\prime}}\right)=(-1)^{j i^{\prime}}, \quad 0 \leq i, i^{\prime}, j, j^{\prime} \leq 1 .
$$

Then $\Omega_{x}$ is a 2-cocycle representing the unique nontrivial cohomology class in $H^{2}\left(L, k^{\times}\right)$. By Theorem 1.1, for any 2-cocycle $\xi$ on $L, \mathcal{M}_{0}(L, 1)$ and $\mathcal{M}_{0}(L, \xi)$ are equivalent as $\mathcal{C}(G, \omega)$-module categories, and therefore so are the corresponding $\mathcal{C}$-module categories $\mathcal{M}(L, 1)$ and $\mathcal{M}(L, \xi)$. This implies that indecomposable $\mathcal{C}$-module categories are in this example parameterized by conjugacy classes of subgroups of $D_{8}$ on which $\omega$ has trivial restriction, as claimed in [2, Section 6.4].

\section{Acknowledgements}

This research was partially supported by CONICET and SeCyT - Universidad Nacional de Córdoba, Argentina.

\section{References}

[1] Etingof P., Gelaki S., Nikshych D., Ostrik V., Tensor categories, Mathematical Surveys and Monographs, Vol. 205, Amer. Math. Soc., Providence, RI, 2015.

[2] Marshall I., Nikshych D., On the Brauer-Picard groups of fusion categories, Math. Z., to appear, arXiv:1603.04318.

[3] Masuoka A., Semisimple Hopf algebras of dimension 6, 8, Israel J. Math. 92 (1995), 361-373.

[4] Masuoka A., Cocycle deformations and Galois objects for some cosemisimple Hopf algebras of finite dimension, in New Trends in Hopf Algebra Theory (La Falda, 1999), Contemp. Math., Vol. 267, Amer. Math. Soc., Providence, RI, 2000, 195-214.

[5] Naidu D., Crossed pointed categories and their equivariantizations, Pacific J. Math. 247 (2010), 477-496, arXiv:1111.5246.

[6] Natale S., On group theoretical Hopf algebras and exact factorizations of finite groups, J. Algebra 270 (2003), 199-211, math.QA/0208054.

[7] Nikshych D., Non-group-theoretical semisimple Hopf algebras from group actions on fusion categories, Selecta Math. (N.S.) 14 (2008), 145-161, arXiv:0712.0585.

[8] Ostrik V., Module categories over the Drinfeld double of a finite group, Int. Math. Res. Not. 2003 (2003), 1507-1520, math.QA/0202130.

[9] Schauenburg P., Hopf bimodules, coquasibialgebras, and an exact sequence of Kac, Adv. Math. 165 (2002), 194-263. 